\title{
Detecção de falhas de alimentação de um motor CC sem escovas via Filtro de Partículas
}

\author{
Letícia M. S. Vianna*, João P. S. Gonçalves**, \\ Iuri A. Monteiro ${ }^{* * *}$, Mateus Giesbrecht ${ }^{* * * *}$ \\ Faculdade de Engenharia Elétrica e de Computação, \\ Universidade Estadual de Campinas - UNICAMP \\ Campinas, SP
*leticiav@dsif.fee.unicamp.br
**jpaulosg@dsif.fee.unicamp.br \\ *** monteiro@dsif.fee.unicamp.br \\ ****mateus@fee.unicamp.br
}

\begin{abstract}
In this article, the problem of fault detection of a brushless dc motor (BLDC) when one of its power suply phases is lost is adressed. This problem has already been solved with different parameter estimation techniques, such as the Least Squares Estimator and the Recursive Least Squares Estimator, in which the parameters of a linear model are estimated, allowing the fault detection. However, this paper proposes the solution by applying the Particle Filter by adding the parameter that indicates the fault in the state vector, transforming the BLDC motor model into a nonlinear one. To validate the method, it was applied to detect the fault of a real BLDC motor and the results were compared with values found in the literature.

Resumo: Neste artigo é tratado o problema da detecção de falhas, quando uma fase de alimentação em um motor de corrente contínua sem escovas (BLDC) é perdida. O problema de detecção de falhas em um motor Brushless DC já foi solucionado por diferentes técnicas de estimação de parâmetros, tal como o Estimador de Mínimos Quadrados e o Estimador de Mínimos Quadrados Recursivos, em que os parâmetros de um modelo linear são estimados, permitindo a detecção da falha. Entretanto, neste artigo é proposta a solução aplicando o Filtro de Partículas ao se colocar o parâmetro que indica a falha no vetor de estado, tornando assim o modelo não linear. Para validar o método, aplicou-se o algoritmo desenvolvido para a detecção de falha de um motor BLDC real e os resultados foram comparados com valores encontrados na literatura.
\end{abstract}

Keywords: Particle filter; parameter estimation; BLDC motor; systems identification; fault detection; fault estimation; Recursive Least Squares.

Palavras-chaves: Filtro de Partículas; estimação de parâmetros; motor BLDC; identificação de sistemas; detecção de falhas; estimativa de falhas; Mínimos Quadrados Recursivo.

\section{INTRODUÇÃO}

Nos últimos anos, a estimação de estados de sistemas lineares com parâmetros incertos tem sido bastante estudada, uma vez que permite a estimação e também a utilização de parâmetros não acessíveis a medições como variáveis de estados (Borges et al., 2008; Xie et al., 2014; Chong et al., 2015).

A fim de se solucionar o problema de estimação de estados, elaboraram-se várias metodologias. Em (Kalman, 1960), é proposto um método de filtragem e predição utilizando processos aleatórios e o método de "transição de estado" de análise de sistemas dinâmicos, método este chamado de

* Os autores agradecem ao apoio financeiro recebido da Coordenação de Aperfeiçoamento de Pessoal de Nível Superior - Brasil (CAPES) - Código de Financiamento 001 e do Conselho Nacional de Desenvolvimento Científico e Tecnológico (CNPq).
Filtro de Kalman. O método é ótimo e, por esse motivo, ainda é muito utilizado para a estimação de sistemas lineares. Em (Nadzilah et al., 2017), é proposto o projeto de um sistema de rastreamento de navios aplicando o algoritmo de Filtro de Kalman. Outra metodologia aplicada é encontrada em (Zhang, 2018), no qual é abordada a estimação de parâmetros pelo método dos Mínimos Quadrados Ordinários e pela aprendizagem utilizando uma Rede Neural Linear.

Esses trabalhos foram desenvolvidos para tratar o caso em que os sistemas são lineares, entretanto os sistemas reais são, em sua maioria, não lineares. Assim, os resultados dos métodos de estimação linear podem falhar quando são utilizados para o processo de estimação de alguns sistemas reais. 
Para lidar com essa questão foram propostos algoritmos aplicáveis aos casos não lineares, tal como o filtro de Kalman Estendido (EKF) (Aguirre, 2007). Esse algoritmo tem sido amplamente utilizado em problemas não lineares, como por exemplo em (Aishwarya and Jayanand, 2016), em que autores propõem o uso do Filtro de Kalman Estendido (EKF) para estimar a velocidade e a posição do rotor de um motor de corrente contínua sem escovas BLDC (do inglês Brushless DC Motor).

Outra das metodologias elaboradas para o tratamento do caso não linear é denominada como Filtro de Partículas, que teve início em 1940 com o trabalho de Metropolis e Norbert Wiener, que sugeriram algo parecido com a filtragem de partículas (Wang et al., 2009). Entretanto, por existirem diversos empecilhos computacionais na época, apenas em 1980 foi possível sua implementação (Simon, 2006).

Os filtros de partículas são geralmente aplicados em problemas de filtragem de sistemas altamente não lineares, no qual o objetivo é estimar os estados e/ou parâmetros desconhecidos de um sistema. São conhecidos por diversos nomes, incluindo Filtro de Monte Carlo, Filtro de Monte Carlo Sequencial (Wang et al., 2009). Esse método é estatístico possuindo um estimador Bayesiano, baseado em probabilidade (Speekenbrink, 2016).

Neste trabalho o algoritmo de filtragem de partículas é apresentado e seus resultados são comparados com os resultados obtidos pelos algoritmos recursivos para o problema de detecção de falhas em um motor de corrente contínua sem escovas. O BLDC é um motor trifásico com comportamento semelhante a de um motor DC, mas com comutação eletrônica. A falha tratada é dada pela perda de uma das fases de alimentação do motor BLDC trifásico.

O problema de detecção de falhas em questão já foi resolvido utilizando um Estimador de Mínimos Quadrados em Batelada por (Tanaka and Giesbrecht, 2018) e também utilizando um Estimador de Mínimos Quadrados Recursivos por (Gonçalves et al., 2018). No entanto, estes métodos apresentam algumas desvantagens: não há como estimar os parâmetros de forma recursiva com o estimador em batelada e mesmo que resolvendo isto com estimador recursivo, a convergência da estimação ainda é lenta para determinadas aplicações. Logo, neste trabalho o problema é resolvido aplicando o Filtro de Partículas. Utilizando o Filtro de Partículas, será possível alcançar uma estimação recursiva e com convergência mais rápida que os algoritmos anteriores, como será mostrado na seção de resultados. O problema da detecção de falha será resolvido com a colocação do parâmetro que indica a falha, que é a resistência equivalente do estator, no vetor de estado. Com isso, o problema de estimação se torna um poblema dual, em que se estimam parâmetros e estados ao mesmo tempo, tornando o um problema de estimação não linear. Daí também a necessidade do uso de um estimador de estados não linear tal qual o Filtro de Partículas.

O trabalho é estruturado da seguinte forma: na seção 2 se apresenta o método de estimação de estados via Filtro de Partículas. Na seção 3, realiza-se a modelagem matemática da máquina BLDC e apresenta-se como este modelo foi utilizado para se estimar o parâmetro físico que indica a falha de alimentação do motor. Na seção 4, apresentam- se as metodologias experimental e computacional para a estimação da resistência e detecção da falha estudada. $\mathrm{Na}$ seção 5, exibem-se os resultados das estimativas dos parâmetros e estados juntamente com a uma análise estatística do modelo. Por fim, na seção 6 são apresentadas as considerações finais e sugestões de futuras pesquisas.

\section{FILTRO DE PARTÍCULAS}

O método de filtragem via Filtro de Partículas faz uso de um estimador baseado em funções de probabilidade: o estimador Bayesiano (Simon, 2006). De acordo com (Gustafsson, 2010), o Filtro de Partículas consiste na repetição de etapas de propagação e correção. A etapa de propagação se refere à geração de partículas com base nas informações que se têm a priori. Essas informações se relacionam à distribuição utilizada para gerar as partículas e resultam na probabilidade a priori.Na etapa de correção, por sua vez, calcula-se a distribuição de probabilidade a posteriori por meio do Teorema de Bayes:

$$
p\left(x_{k} \mid Y_{k}\right)=\frac{p\left(x_{k} \cap Y_{k}\right)}{p\left(Y_{k}\right)},
$$

isto é, a probabilidade condicional de $x_{k}$, dadas as observações $Y_{k}$ até o instante $k$, é igual à razão entre a probabilidade da interseção entre $x_{k}$ e $Y_{k}$ e a probabilidade de $Y_{k}$.

Por fim, atribui-se, a cada uma das partículas, um peso como medida de verossimilhança.

Seja o seguinte modelo não linear no espaço de estado:

$$
\begin{aligned}
x_{k+1} & =f_{k}\left(x_{k}, u_{k}, \eta_{k}\right), \\
y_{k} & =h_{k}\left(x_{k}, \nu_{k}\right),
\end{aligned}
$$

em que $k$ é o índice temporal; $x \in \mathbb{R}^{n}, y \in \mathbb{R}^{l}, u \in \mathbb{R}^{m}$ são vetores de estados, observações e entradas, respectivamente, com as correspondentes funções de evolução de estado $f_{k}$ e de observação $h_{k}$. O vetor $\eta_{k}$ é o ruído de processo e $\nu_{k}$, o vetor de ruído de observação. Supõe-se ainda que os ruídos são independentes e identicamente distribuídos (i.i.d.) e que possuem funções de densidade de probabilidade conhecidas. As funções $f_{k}(\cdot)$ e $h_{k}(\cdot)$ são vetores de equações variantes no tempo e não lineares que representam o sistema.

A filtragem de partículas se inicia na geração de um conjunto de $N$ amostras aleatórias para a função de densidade de probabilidade (pdf, do inglês probability density function) a posteriori, $p\left(x_{k-1} \mid y_{k-1}\right)$, em que $\left\{x_{k-1}^{i}\right\}_{i=1}^{N}$ são representações das anteriormente mencionadas partículas. Para o instante de tempo inicial, $k=0$, supõe-se que a pdf é conhecida, $p\left(x_{0}\right)=p\left(x_{0} \mid y_{0}\right)$, e que não é condicionada à observação neste instante de tempo. A quantidade $N$ de partículas é escolhida pelo projetista e sua escolha leva em consideração a exatidão e o esforço computacional desejados (Wang et al., 2009).

A partir da pdf a posteriori, propagam-se as partículas conforme a equação de transição de estado (2). Assim, as partículas a priori do próximo instante de tempo, $\left\{x_{k}^{i}\right\}_{i=1}^{N}$, são obtidas. Esse procedimento produz um conjunto de amostras a priori da pdf $p\left(x_{k} \mid y_{k-1}\right)$.

Na etapa de correção, calcula-se o peso $q_{k}^{i}$ para cada uma das partículas. Esse peso é uma medida da probabilidade 
da observação da saída para o valor do estado a priori, $q_{k}^{i}=p\left(y_{k} \mid x_{k}^{i}\right)$, sendo calculado por

$$
q_{k}^{i}=\frac{1}{(2 \pi)^{l / 2}|R|^{0,5}} \exp \left(\frac{-\left[y^{*}-\bar{y}\right]^{T} R^{-1}\left[y^{*}-\bar{y}\right]}{2}\right),
$$

em que $i=1, \ldots, N$ e é utilizada a equação de observação (3) para se ter os valores estimados de saídas $\bar{y}$. Os parâmetros dados pela pdf gaussiana são

$$
p\left(y_{k} \mid x_{k}, \nu_{k}\right)=\mathcal{N} \sim(\bar{y}, R),
$$

em que $R$ é a matriz de covariância do ruído de medição; $\vec{y}$ é a observação estimada a priori; e $y^{*}$ é a observação real, e $\mathcal{N}(\cdot, \cdot)$ denota uma pdf gaussiana (Simon, 2006).

Em seguida, normalizam-se os pesos por meio da relação

$$
q_{k}^{i}=\frac{q_{k}^{i}}{\sum_{j=1}^{N} q_{k}^{j}}
$$

Um conjunto de partículas a posteriori, $\left\{x_{k}^{i}\right\}_{i=1}^{N}$, é gerado. Com base nos pesos normalizados, as partículas são reamostradas por meio da função de densidade acumulada, que serve como parâmetro na seleção das partículas mais significativas - isto é, com maior peso e que, portanto, fornecem maior verossimilhança - e são utilizadas para gerar o novo conjunto da pdf desejada $p\left(x_{k} \mid y_{k}\right)$. Finalizase, assim, um ciclo do algoritmo, o qual se repete até $k$ iterações - número de amostras - sejam concluídas.

Este algoritmo recebe o nome de Filtro de Partículas SIS (do inglês, Sampling-Importance-Resampling) e utiliza a amostragem por importância para selecionar as melhores partículas e então replicá-las (Salmond and Gordon, 2005).

\section{MODELAGEM DO MOTOR BRUSHLESS DC}

O motor BLDC, é trifásico, sendo que seu acionamento e controle é realizado por um drive eletrônico (ex. inversor por modulação de largura de pulso (pwm, do inglês Pulse Width Modulation). No entanto, seu comportamento dinâmico por fase é semelhante a um motor CC com escovas. A principal diferença é que o torque eletromagnético no BLDC irá depender de todas as 3 forças contraeletromotrizes defasadas. Considerando tal fato, optou-se por utilizar um modelo dinâmico simplificado do BLDC em que seu comportamento pudesse ser modelado idêntico a um motor CC com escovas, facilitando assim tanto a estimação quanto o aparato experimental. O modelo considerado é do tipo MISO (multiple-input single-output) a tempo contínuo, em que a saída é a corrente de armadura e as entradas são a velocidade do eixo e a tensão de fase, sendo descrito por:

$$
\frac{d i_{a}}{d_{t}}=-\frac{R_{a}}{L} i_{a}-\frac{k_{e}}{L} \omega_{m}+\frac{1}{L} V,
$$

em que $R_{a}$ é a resistência elétrica equivalente, $L$ é a indutância equivalente, $k_{e}$ é a constante elétrica, todas em unidades do sistema internacional (SI). As variáveis $i_{a}, \omega_{m}$ e $V$ são corrente de armadura, velocidade do eixo e tensão de fase, respectivamente.

Para a estimação, o modelo foi dicretizado utilizando uma aproximação de Euler para a derivada, resultando em

$$
\begin{aligned}
i_{a k+1} & =\theta_{1} i_{a k}+\theta_{2} \omega_{m k}+\theta_{3} V_{k}, \\
\theta_{1} & =\left(1-\frac{R_{a} \Delta_{t}}{L}\right), \\
\theta_{2} & =-\frac{k_{e} \Delta_{t}}{L}, \\
\theta_{3} & =\frac{\Delta_{t}}{L},
\end{aligned}
$$

em que $\Delta_{t}$ é o período de amostragem.

O modelo apresentado na equação 8 é linear se os parâmetros forem conhecidos, mas como deseja-se estimar a resistência do estator $R_{a}$ que é um dos indicadores da falha de perda de uma das fases, esse parâmetro pode ser adicionado ao vetor do estado e o modelo torna-se não linear. Logo, inserindo a resistência no vetor de estados $x_{k}$, tem-se o seguinte modelo em espaço de estado não linear

$$
\begin{aligned}
& x_{k+1}= {\left[\begin{array}{cr}
\left.1-\frac{x_{2_{k}} \Delta_{t}}{L}\right) & 0 \\
0 & 1
\end{array}\right] x_{k}+} \\
&+ {\left[\begin{array}{cc}
-\frac{k_{e} \Delta_{t}}{L} & \frac{\Delta_{t}}{L} \\
0 & 0
\end{array}\right]\left[\begin{array}{c}
\omega_{m_{k}} \\
V_{k}
\end{array}\right], } \\
& y_{k}=\left[\begin{array}{ll}
1 & 0
\end{array}\right] x_{k},
\end{aligned}
$$

em que o vetor de estado é $x_{k}=\left[\begin{array}{ll}x_{1_{k}} & x_{2_{k}}\end{array}\right]^{T}=\left[\begin{array}{ll}i_{a_{k}} & R_{a_{k}}\end{array}\right]^{T}$. Assim, tem-se a representação do motor BLDC no espaço de estado, dada por:

$$
\begin{aligned}
x_{k+1} & =f_{k}\left(x_{k}, u_{k}\right), \\
y_{k} & =C x_{k},
\end{aligned}
$$

em que

$$
f_{k}\left(x_{k}, u_{k}\right)=\left[\frac{\frac{L-R_{a k} \Delta_{t}}{L} i_{a k}-\frac{k_{e} \Delta_{t}}{L} \omega_{m_{k}}+\frac{\Delta_{t}}{L} V_{k}}{R_{a k}}\right]
$$

é a função não linear de evolução de estados, com

$$
u_{k}=\left[\begin{array}{ll}
\omega_{m_{k}} & V_{k}
\end{array}\right]^{\mathrm{T}}, \quad y_{k}=\left[i_{a k}\right]
$$

e

$$
C=[10]
$$

a matriz de saída do sistema.

Os parâmetros $L$ e $k_{e}$ foram retirados de (Gonçalves et al., 2018), em que foram determinados por meio do Estimador de Mínimos Quadrados em Batelada (Aguirre, 2007).

A partir disso, o Filtro de Partículas é aplicado para a estimação dos estados do modelo não linear; Com isso, espera-se que na ocorrência de uma falha o valor da resistência do estator mude e que essa mudança seja estimada pelo filtro.

\section{METODOLOGIA}

\subsection{Metodologia Experimental}

Os dados foram coletados utilizando o software Labview e um aquisitor de dados (DAQ, do inglês Data Acquisition) modelo NI USB-6008. Os sensores para medição de tensão e corrente foram LV-20P e LA-55P e um sensor hall foi utilizado para medir a velocidade. Uma placa para condicionamento de sinais foi projeta por (da Silva, 2017) para captar os sinais de corrente e tensão de linha do motor. O acionamento do motor é feito por instrumentos 
virtuais desenvolvidos no Labview e a falha foi simulada retirando uma das fases do motor BLDC.

No experimento foram coletadas as seguintes variáveis: a tensão entre duas fases (tensão de linha), a velocidade $\omega_{m}$ e a corrente de armadura $i_{a}$ de uma das fases que não fosse a fase de falha. A tensão de fase $V$ foi obtida dividindo a tensão de linha por $\sqrt{3}$. Utilizou-se uma taxa de amostragem de $10 \mathrm{~Hz}$ (período de amostragem de $0,1 \mathrm{~s}$ ), a qual mostrou-se suficiente para o trabalho.

Os testes no motor foram inicializados quando o mesmo chegava ao regime e, então a falha era introduzida. Assim, após cerca de 20 minutos ligado, a temperatura do motor estabilizava e uma das fases era retirada por cerca de 5 minutos. As entradas, em cada uma das fases, foram sinais binários pseudo aleatório (PRBS). A falha ocorre aproximadamente na amostra 3000. Maiores informações sobre a parte experimental podem ser encontradas em (Gonçalves et al., 2018) e (da Silva, 2017).

\subsection{Metodologia Computacional}

Seja a representação do motor BLDC no espaço de estado

$$
\begin{aligned}
x_{k+1} & =f_{k}\left(x_{k}, u_{k}\right), \\
y_{k} & =C x_{k} .
\end{aligned}
$$

A estimação de estados, e consequentemente da resistência $R_{a}$ se deu por meio do algoritmo do Filtro de Partículas descrito na seção 2. Na implementação deste trabalho, utilizou-se o método de reamostragem multinomial para a seleção das partículas mais significativas (Simon, 2006). Nesta técnica de reamostragem, as partículas do conjunto inicial com pequena variância de pesos são mais prováveis de serem descartadas, enquanto que as de alto peso são replicadas (Candy, 2009).

Os estados iniciais foram supostos distribuídos conforme a distribuição gaussiana $p\left(x_{0}\right)=\mathcal{N} \sim(0 ; 0,09)$ e foram feitas simulações para diferentes quantidades de partículas. Os dados de entrada e saída utilizados foram amostrados com frequência de $10 \mathrm{~Hz}$ e são apresentados em (Gonçalves et al., 2018).

É importante ressaltar que durante a estimação, na equação de evolução de estados, foram adicionados ao estado estimado ruídos de processo com distribuição gaussiana. Estes ruídos têm como objetivo representar as incertezas existentes no modelo, entretanto na equação de observação não é necessário a adição de ruídos, uma vez que os dados coletados já possuem incertezas.

\section{RESULTADOS}

Os resultados obtidos para a resistência de armadura $R_{a}$ e para a estimação da corrente $i_{a}$ foram analisados e comparados com os resultados encontrados em (Gonçalves et al., 2018). Na tabela 1 são apresentados os Erros Quadráticos Médios (EQM) calculados para o sinal de corrente estimado com a execução do Filtro do Partículas com diferentes números $N$ de partículas. Da tabela é observado que um aumento no número das partículas utilizadas está diretamente relacionado a um aumento da exatidão mas também a um aumento no tempo de execução do algoritmo.
Tabela 1. Erro Quadrático Médio para diferentes números de partículas.

\begin{tabular}{ccc}
\hline $\mathrm{N}$ & EQM de $I_{a}(\mathrm{~A})$ & Tempo de execução (s) \\
\hline 100 & 0,0249 & 22,8501 \\
500 & 0,0207 & 153,2474 \\
1000 & 0,0200 & 255,4529 \\
5000 & 0,0196 & 1480,2007 \\
\hline
\end{tabular}

Tabela 2. Valores médios do parâmetro $R_{a}$ estimado pelo Filtro de Partícula para diferentes números de partículas, antes e depois da falha.

\begin{tabular}{ccc}
\hline $\mathrm{N}$ & $\bar{R}_{a_{S F}}(\Omega)$ & $\bar{R}_{a_{C F}}(\Omega)$ \\
\hline 100 & 1,2551 & 1,1373 \\
500 & 1,2541 & 1,1328 \\
1000 & 1,2537 & 1,1322 \\
5000 & 1,2530 & 1,1320 \\
\hline
\end{tabular}

Tabela 3. Valores do parâmetro $R_{a}$ estimado pelo Estimador de Mínimos Quadrados em Batelada (Gonçalves et al., 2018), antes e depois da falha.

\begin{tabular}{cc}
\hline$R_{a_{S F}}(\Omega)$ & $R_{a_{C F}}(\Omega)$ \\
\hline 1,2530 & 1,1307 \\
\hline
\end{tabular}

Na tabela 2 são apresentados os valores médios da resistência $R_{a}$ estimada pela filtragem de partículas, em que $\bar{R}_{a_{S F}}$ representa a média da resistência estimada antes da falha e $\bar{R}_{a_{C F}}$ a média da resistência estimada depois da ocorrência da falha. Já na tabela 3, os valores apresentados são os obtidos pelo Estimador de Mínimos Quadrados em Batelada em (Gonçalves et al., 2018), em que $R_{a_{S F}}$ representa a resistência estimada antes da falha e $R_{a_{C F}}$ a resistência estimada depois da falha. Comparando os valores das tabelas 2 e 3 é verificado que os valores estimados de $R_{a}$ são aproximadamente iguais para ambas as técnicas de estimação.

Na figura 1 são apresentados os resultados da estimação da resistência de armadura $R_{a}$ com um filtro com $N=500$ partículas em comparação com os valores obtidos por batelada em (Gonçalves et al., 2018). No teste apresentado, a falha foi introduzida aproximadamente no instante $k=$ 3000s. Da figura, visualiza-se o momento em que ocorreu a falha através da variação da resistência de armadura estimada, indicando que o filtro foi capaz de detectá-la e rapidamente convergir para o valor de resistência estimado em batelada para o caso com falha.

Na figura 2 é apresentada a estimação da corrente de armadura $i_{a}$ do filtro com $N=500$ partículas em comparação com a corrente medida. É possível observar que os valores estimados são próximos dos medidos e que a falha ocorre em aproximadamente $k=3000$ s.

Por fim, na figura 3 são apresentados os erros de estimação do estado $i_{a}$ e do parâmetro $R_{a}$. Verifica-se que são próximos de zero. Nota-se também que, no instante da falha, o erro para o parâmetro $R_{a}$ aumenta, mas que em poucos instantes de tempo retorna a zero, indicando a convergência do filtro. Vale observar que a velocidade de convergência do Filtro de Partículas foi maior que 


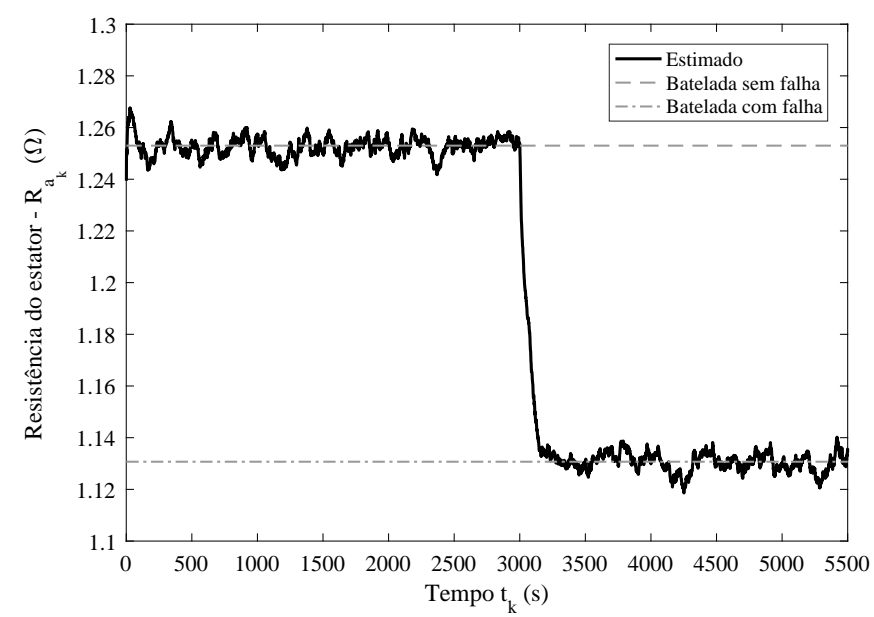

Figura 1. Estimação da resistência do estator. As curvas contínuas, em preto, representam os valores encontrados pelo Estimador de Mínimos quadrados em Batelada e as curvas pontilhadas, em cinza, os valores estimados.

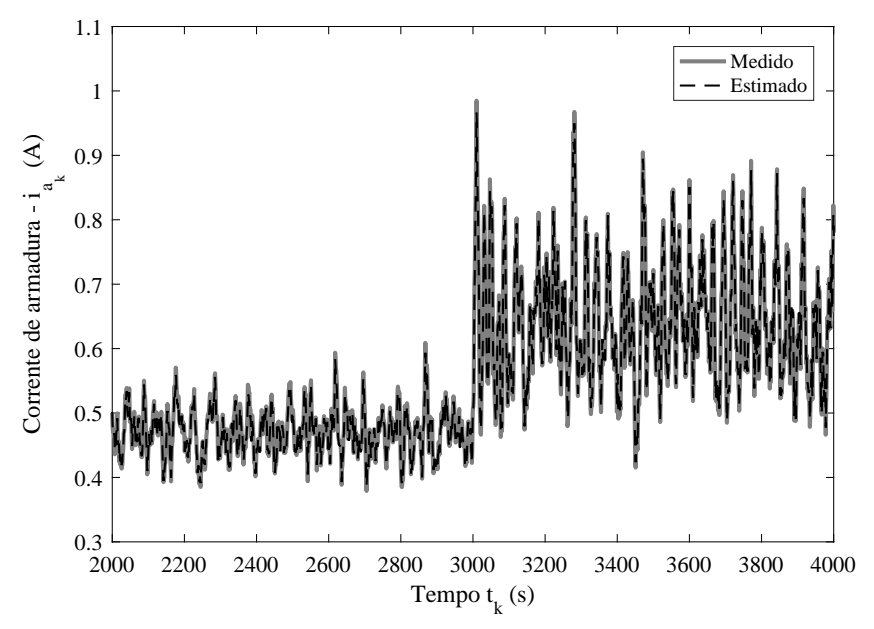

Figura 2. Estimação da corrente de armadura. As curvas contínuas, em preto, representam os valores medidos e as curvas pontilhadas, em cinza, os valores estimados.

a convergência do MQR mostrada em Gonçalves et al. (2018).

\section{CONCLUSÃO}

Neste trabalho foi proposta a aplicação do Filtro de Partículas como método de estimação de parâmetros de um motor BLDC. O Filtro de Partículas aplicado na presença de falhas apresentou bom desempenho tanto na estimação do estado, quanto na estimação do parâmetro desconhecido, se comparado aos resultados obtidos pelo Estimador de Mínimos Quadrados em Batelada. Além disso, ele convergiu com uma velocidade consideravelmente maior que o Mínimos Quadrados Recursivo. Desse modo, verificou-se a eficiência do método utilizado por meio de experimentos computacionais.

Para trabalhos futuros, será proposta uma representação matemática do modelo que englobe os parâmetros mecâni-
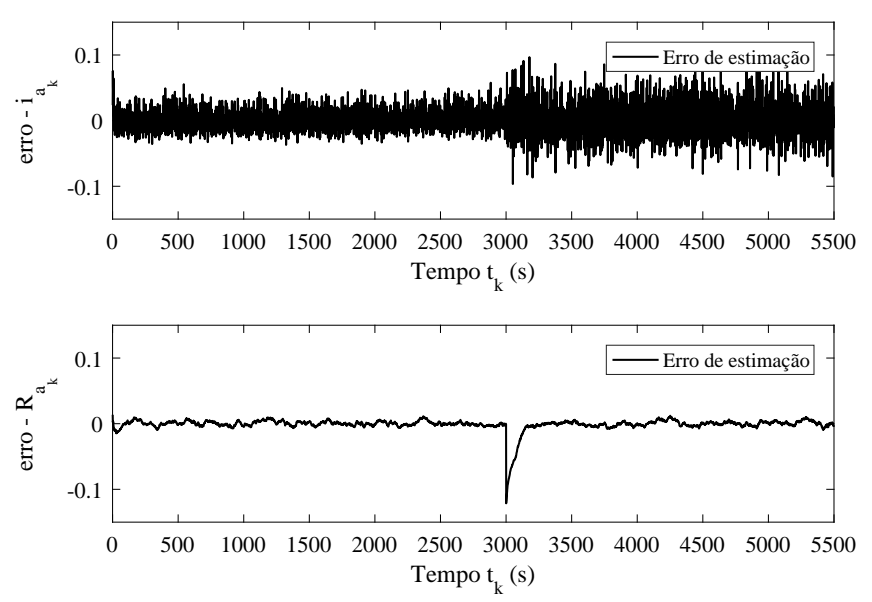

Figura 3. Erros de estimativas. Na figura superior, o erro de estimação da corrente de armadura e a figura inferior o erro da estimação da resistência do estator. As curvas, em preto, representam os valores de erros encontrados.

cos do motor BLDC, tornando-o mais próximo do sistema real.

\section{REFERENCIAS}

Aguirre, L.A. (2007). Introdução à identificação de sistemas-Técnicas lineares e não-lineares aplicadas a sistemas reais. Editora UFMG, 3 edition.

Aishwarya, V. and Jayanand, B. (2016). Estimation and control of sensorless brushless dc motor drive using extended kalman filter. In 2016 International Conference on Circuit, Power and Computing Technologies (ICCPCT), 1-7. IEEE.

Borges, R.A., Montagner, V.F., Oliveira, R.C., Peres, P.L.D., and Bliman, P.A. (2008). Parameter-dependent $\mathcal{H}_{2}$ and $\mathcal{H}_{\infty}$ filter design for linear systems with arbitrarily time-varying parameters in polytopic domains. Signal Processing, 88(7), 1801-1816.

Candy, J.V. (2009). Bayesian Signal Processing: Classical, Modern, and Particle Filtering Methods. John Wiley \& Sons.

Chong, M.S., Nešić, D., Postoyan, R., and Kuhlmann, L. (2015). Parameter and state estimation of nonlinear systems using a multi-observer under the supervisory framework. IEEE Transactions on Automatic Control, $60(9), 2336-2349$.

da Silva, R.D. (2017). Identificação de parâmetros e projeto do sistema de controle de velocidade de motores de corrente contínua sem escovas bldc. Trabalho de Conclusão de Curso(Bacharel em Engenharia de Controle e Automação), UNESP (Universidade Estadual Paulista), Sorocaba, Brasil.

Gonçalves, J.P.S., Tanaka, V.S., and Giesbrecht, M. (2018). Aplicação de identificação caixa cinza e estimação recursiva na detecção de falha de um motor cc sem escovas. XXII Congresso Brasileiro de Automática.

Gustafsson, F. (2010). Particle filter theory and practice with positioning applications. IEEE Aerospace and Electronic Systems Magazine, 25(7), 53-82.

Kalman, R.E. (1960). A new approach to linear filtering and prediction problems. Transactions of the ASMEJournal of Basic Engineering. 
Nadzilah, A., Gandana, D.M., Muliadi, J., and Daryanto, Y. (2017). Application of Kalman filter to track ship Maneuver. In Cyber and IT Service Management (CITSM), 2017 5th International Conference on, 1-5. IEEE.

Salmond, D. and Gordon, N. (2005). An introduction to particle filters. State space and unobserved component models theory and applications, 1-19.

Simon, D. (2006). Optimal state estimation: Kalman, $\mathcal{H}_{\infty}$, and nonlinear approaches. John Wiley \& Sons.

Speekenbrink, M. (2016). A tutorial on particle filters. Journal of Mathematical Psychology, 73, 140-152.

Tanaka, V.S. and Giesbrecht, M. (2018). Análise e diagnóstico de falha em motor brushless dc. In VIII ENAM - Encontro Nacional de Máquinas Rotativas, 2018, Rio de Janeiro. Anais do VIII ENAM.

Wang, D., Zhao, J., and Wang, W. (2009). Particle filter based robust mobile robot localization. IEEE International Conference on Mechatronics and Automation, 817821.

Xie, X.P., Yang, D.S., and Zhu, X.L. (2014). Relaxed observer design of discrete-time $\mathrm{T}-\mathrm{S}$ fuzzy systems via a novel multi-instant fuzzy observer. Signal Processing, 102, 296-303.

Zhang, W. (2018). System identification of linear systems with a linear neural network:- on relationship of lms learning and least squares estimation. In 2018 Chinese Control And Decision Conference (CCDC), 4615-4620. IEEE. 